\title{
TECHNIQUES TO REDUCE THE COMPUTATIONAL COST OF FINITE ELEMENT MODELS OF THE LOWER LIMB MUSCLES
}

\author{
E. ELYASI ${ }^{1}$, A. PERRIER ${ }^{1,2}$, M. BAILET ${ }^{3}$, Y. PAYAN ${ }^{1}$ \\ ${ }^{1}$ Univ. Grenoble Alpes \\ CNRS, TIMC-IMAG, 38000 Grenoble, France \\ elyasi.elaheh@gmail.com \\ ${ }^{2}$ Groupe hospitalier Diaconesses-Croix Saint-Simon, \\ 75020 Paris, France \\ ${ }^{3}$ TwInsight, \\ 38000 Grenoble, France
}

Keywords: Combined FEM-multibody modeling, Mesh embedding.

\begin{abstract}
Modeling the lower limb muscles using the Finite Element (FE) method is required for various applications including injury mechanisms or when stress/strain distribution in the muscle is of interest. When the muscles are represented with 3D FE models, the interaction between the muscles can be taken into account that has an effect on their force production. However, the computational cost of such a method is considerably high. Besides, in many cases, a major part of this computational cost is committed to gain unnecessary information. For instance, when having two FE muscles in contact, both muscles need to get finely meshed to conserve their surface details even if having the stress/strain distribution inside one of the two muscles is not required. As a result, the current study aims to explore a model reduction technique based on mesh embedding to decrease the computational cost of such models. A combination of Computerized Tomography and Magnetic Resonance Imaging (MRI) data obtained from a volunteer subject was used to generate a musculoskeletal model of the quadriceps muscle group. The modeling process was performed in ArtiSynth which is an opensource 3D modeling platform supporting combined simulation of multibody and finite element models. This platform allows the attachment of a passive embedded mesh to a FE body so that it deforms in accordance with the motion of the FE body. Considering that the external forces applied to the passive mesh can be propagated back to the FE body attached to it, contact can be defined between the embedded mesh and any other structure. A full and a reduced model are generated and are used to simulate a passive deep knee flexion to test the reliability of the method. The kinematic outcomes are compared against data points obtained from MRI at different flexion angles. The results show that the proposed methodology can be considered as a substitute to fully FE models without a substantial sacrifice on the outcomes despite having lower computational cost.
\end{abstract}

\section{INTRODUCTION}

Using the Finite Element (FE) method for modeling the muscles of the lower limb can be 
beneficial in applications such as injury mechanisms or when stress/strain distribution in the muscle is of interest [1-4]. Having a 3D FE representation of the muscles provides the opportunity to model them in interaction with an environment of other muscles, connective tissue, and bones and not as isolated units that only transmit axial forces to bones via tendons. The surrounding tissues in the muscle's environment can transfer transversal forces to the muscle tissue. These transversal forces have been shown to result in decreasing muscle force production [5]. Given that the lower limb consists of some of the largest skeletal muscles of the body, the aforementioned effect could be important in its muscular architecture.

However, musculoskeletal (MS) models with 3D FE muscles suffer from having a high preparation time as well as a high computational cost due to the number of elements and complex contact between different muscles. This limitation acts as a major barrier to use them for further applications and especially for dynamic simulations. Furthermore, these models are rarely used for muscle recruitment problems due to the excessive computational burden.

As a result, reducing this time seems to be a big improvement. In this sense, the objective of the current study is to explore the use of a model reduction technique on the MS model of the lower limb that includes the 3D FE representation of the quadriceps muscle group. This method can provide a simplified version of the model and we control the loss of accuracy that it can cause in comparison with the full model (that includes nonlinear hyperelastic material models with complex contacts between muscles).

The proposed technique relies on mesh embedding that is a well-known approach in the concept behind this attached to an underly

the global deformation of

the number of dynamic

the embedding FE grid.
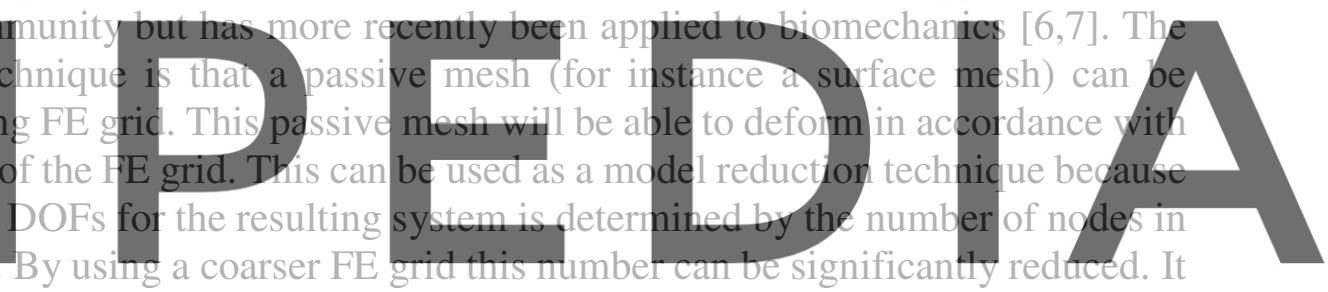

is important to be mentioned that this technique can handle contact between the passive surface

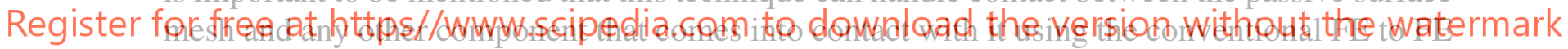

contact detection methods. When the passive mesh comes into contact with another component, the contact force will be simply propagated back to the FE grid and deform the FE grid accordingly.

In the following, the MS model of the lower limb with 3D FE representation of the quadriceps muscles is first described and used to simulate passive deep knee flexion. Subsequently, the mesh embedding technique is explained and gets evaluated on this FE model to see if this technique can be used to reduce the computational cost by replacing the surrounding muscles to the Rectus Femoris (RF) muscle with embedded surfaces without a big compromise on the quality of the results. The kinematic outcomes of the models during passive deep knee flexion get compared against Magnetic Resonance Imaging (MRI) data obtained at different flexion angles for validation purposes.

\section{METHODS}

As the first step of the study, an MS model of the lower limb in presence of the quadriceps muscle group represented as 3D FE components is generated to simulate passive deep knee flexion. The motion of the tibiofemoral joint is controlled using the MRI data of a subject 
collected at various knee flexion angles and the simulation gets validated by comparison of the kinematic outcomes of the patellofemoral joint with the MRI-based kinematic data points. A mesh embedding technique is used on the 3D FE muscle components to get evaluated as a method to reduce the computational cost of the model. The modeling is conducted in Artisynth that is an open-source Java-based modeling platform that allows the combination of the FE method with multibody modeling techniques while supporting contact and constraints [8] as well as embedded meshes [7] (www.artisynth.org).

\subsection{Geometry reconstruction}

A healthy subject (male, 40 years old, $94 \mathrm{~kg}, 1.73 \mathrm{~m}$ ) was volunteered and gave his informed consent for participating in the experimental data collection that was part of a pilot study approved by an ethical committee (MammoBio MAP-VS pilot study, IRMaGe platform, Univ. Grenoble Alpes). The subject underwent a total of 5 sessions of non-weight-bearing MRI using a clinical MRI system (Achieva 3.0T dStream Philips Healthcare) and one session of computed tomography (CT) scan.

The CT scan was taken to capture the surfaces of the bones precisely to be used as a reference for registering the bones segmented from different MRI sets. The first MRI session was performed to obtain the full geometry of the lower limb muscles while the subject was lying still in the supine position $\left(6.99 \times 0.97 \times 0.97 \mathrm{~mm}^{3}\right.$ intervals respectively in the axial, sagittal, and coronal views). The second MRI was obtained from the subject's right knee to capture the

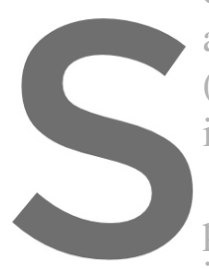
articulation, the connective $\left(0.31 \times 0.7 \times 0.31 \mathrm{~mm}^{3}\right.$ intervals in the supine position with an

The third to fifth MRI sessions were passive deep knee flexion as we
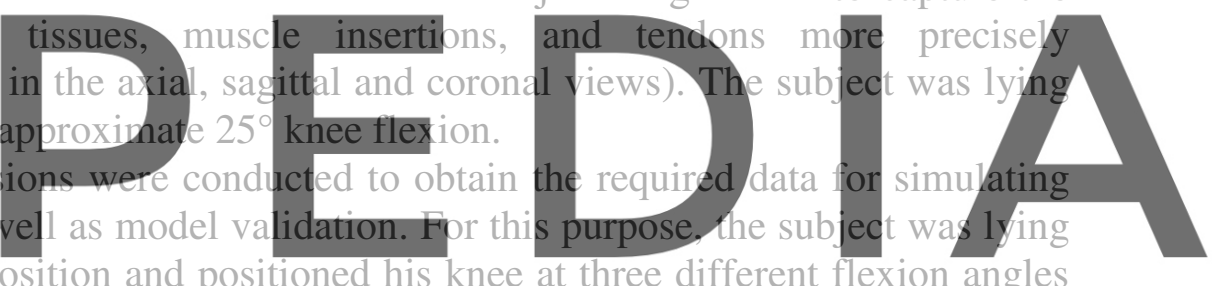
in the left lateral recumbent position and positioned his knee at three different flexion angles

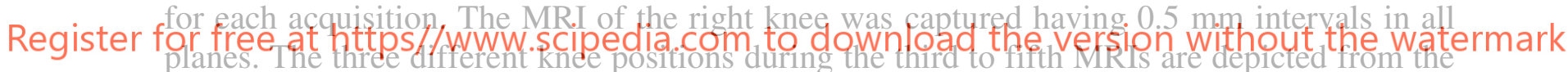
sagittal view in Figure 1. The knee was flexed at 55.6 , 99.6 and $137.5^{\circ}$ respectively during the first to the third MRI. Manual segmentation was performed on all the images of the subject in Amira 6.5.0 in order to prepare the model geometry.
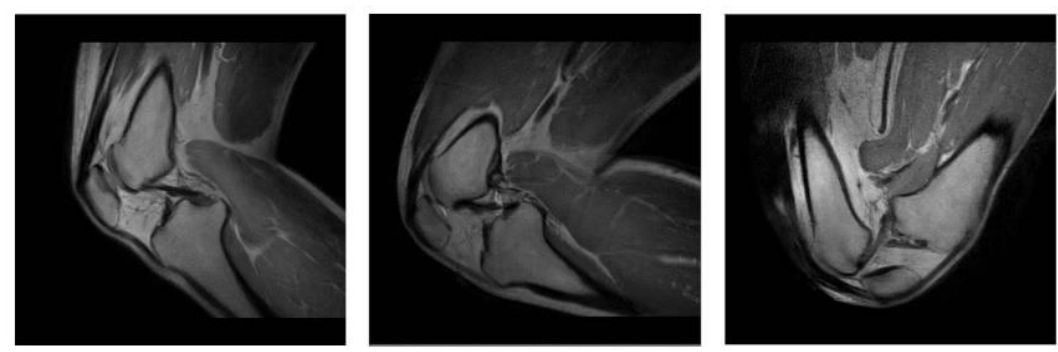

Figure 1: The sagittal view of the right knee MRI at three different flexion angles.

\subsection{Mesh generation}

The FE model of the quadriceps muscle group including Rectus Femoris (RF), Vastus 
Medialis (VM), Vastus Lateralis (VL), Vastus Intermedius (VI), and the quadriceps tendon (QT) was generated using the muscle geometries reconstructed from the MRI segmentation (Figure 2). In order to control the number of finite elements and in particular to avoid very small elements, the segmented muscle surfaces were smoothed to remove the unnecessary surface details and spikes. The end regions where the muscles insert into the bone were also simplified to avoid very small elements and to facilitate the meshing process with methods such as solid map meshing.

The process of mesh generation was conducted in HyperMesh 2019 (Altair Engineering, Inc., USA) to generate hexahedral dominant meshes (with control of element qualities and a limited number of wedge elements) for the four muscle parts as well as for the QT. The maximum element size was approximately $6 \mathrm{~mm}$ to mesh the muscle parts and resulted in 2100 elements for RF, 1325 for VI, 9290 for VL, and 2308 for VM. For the Quadriceps Tendon (QT), the approximate maximum element size was $3 \mathrm{~mm}$ which resulted in a mesh with 5200 elements.
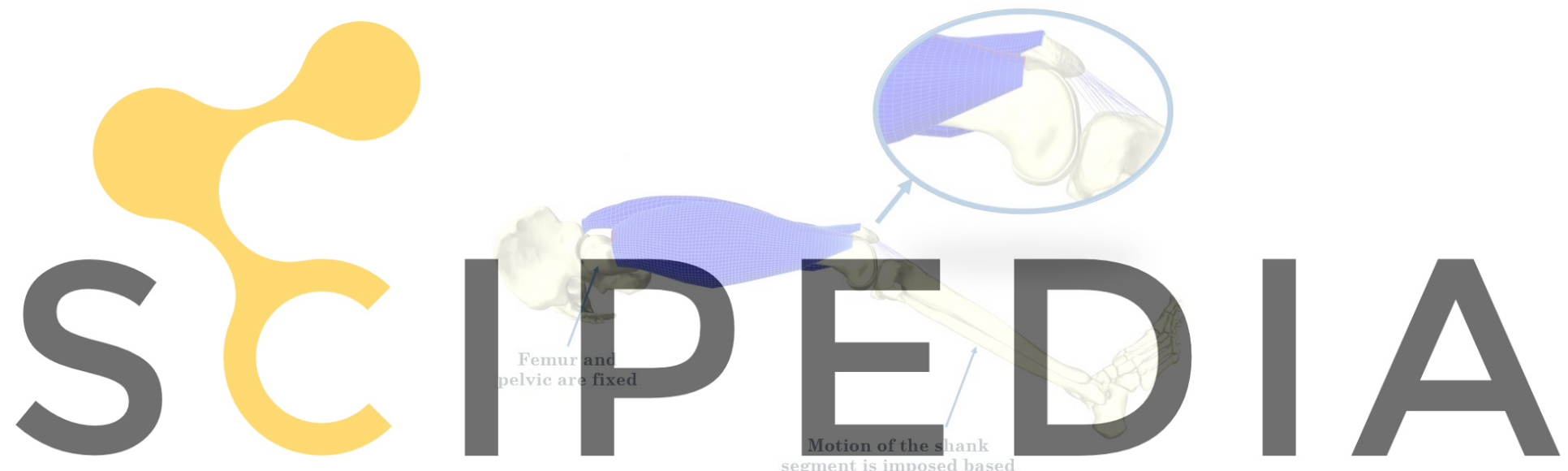

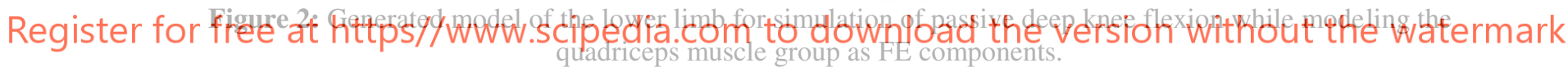

\subsection{Using embedded surface mesh for the quadriceps muscie group}

In order to explore the application of the mesh embedding technique on the generated model, we assume that the RF muscle and QT to be the most important components that we want to monitor in terms of stress distribution. The other muscles (VI, VL, and VM) are the surrounding muscles of our main muscle and their stress distribution is assumed not to be a priority. Thus we will be replacing these muscles with embedded surfaces in a coarse FE grid to reduce the overall computational cost.

To describe the further steps of this reduced model, it is first required to explain the mechanism of "attachment" in the Artisynth platform [7], which enables us to connect surface mesh voxels to a deformable FE body.

The attachment mechanism works by defining the coordinates of the attached component $\left(x_{a}\right)$ to be a function of the coordinates of one or more master components to which it is attached $\left(x_{m}\right)$ as follows:

$$
x_{a}=f\left(x_{m}\right)
$$


This implies that the velocities of these components have a linear relationship similar to:

$$
\dot{\mathrm{x}}_{\mathrm{a}}=\mathrm{G}_{\mathrm{am}}\left(\dot{\mathrm{x}}_{\mathrm{m}}\right), \quad \mathrm{G}_{\mathrm{am}} \equiv \nabla \mathrm{f}\left(\mathrm{x}_{\mathrm{m}}\right)
$$

Based on the principle of virtual work, the force $\left(f_{a}\right)$ of the attached point will be able to propagate back to the master components $\left(f_{m}\right)$ as follows:

$$
\mathrm{f}_{\mathrm{m}}=\mathrm{G}_{\mathrm{am}}^{\mathrm{T}} \mathrm{f}_{\mathrm{a}}
$$

Now with this background, for a point attached to a FEM (for instance each vertex of the embedded surface mesh that is attached to a FEM grid), its position and velocity is a weighted sum of nearby nodal positions $\boldsymbol{x}_{\boldsymbol{j}}$ as follows:

$$
x_{a}=\sum_{j} w_{j} x_{j}, \quad \dot{x}_{a}=\sum_{j} w_{j} \dot{x}_{j}
$$

Forces applied to each vertex $\left(f_{a}\right)$ then propagate back to each node according to:

$$
f_{j}=w_{j} f_{a}
$$

Given this information, to apply the technique to the VI, VM, and VL muscles, a coarse FEM grid was generated for these muscles to embed their surface meshes. An example of the embedded mesh in comparison to the normal FE mesh is depicted for the VI muscle in Figure 3. It is important to mention that the embedded surface mesh does not require a high level of treatment and simplification of the insertion sites. This significantly reduced the preparation time of the model. Figure 4 illustrates the model having the RF and QT as finely meshdd components and the other mus
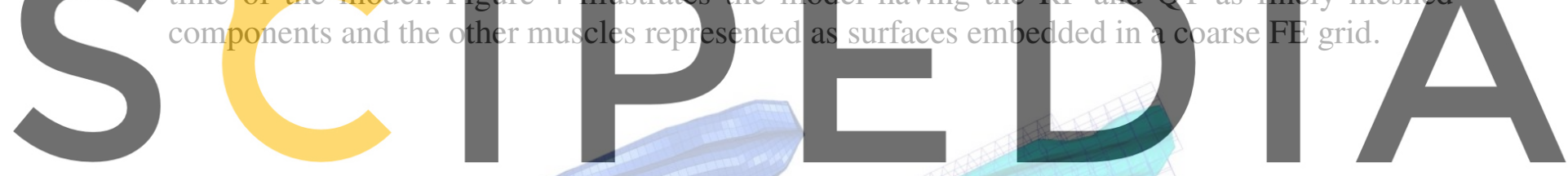

Register for free at https//www.scipedia.com to download the version without the watermark

Figure 3: The image on the left is the hexahedral dominant mesh with 1325 elements, generated for the VI muscle and used in the finely-meshed FE model. The image on the right is the polygonal surface mesh embedded in a coarse FE mesh composed of 324 voxel elements of a maximum of $2 \mathrm{~cm}$ edges.

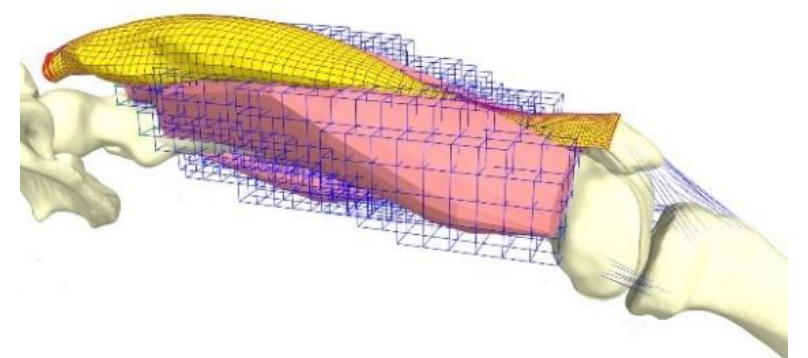

Figure 4: Generated lower limb model while considering the RF and QT as main FE components that are finely meshed (highlighted in yellow). The other muscles are modeled as surface meshes embedded in FE grids. 


\subsection{Material Properties}

The muscle normally experiences large displacements and strains and has a nonlinear stress/strain curve. As a result, when the muscle is in the passive state and has no activation, its behavior can be best represented by using a hyperelastic material model [9]. In the passive deep flexion simulation of the current study, the focus is mainly put on the overall behavior of the muscles and the patellofemoral kinematics. Therefore, we have assumed that the passive muscle behavior can be modeled with a nearly incompressible isotropic neo-Hookean hyperelastic material model. The parameters used for the quadriceps muscles were defined based on the findings of Affagard et al. who presented an in vivo method to identify the behavior of the thigh muscles based on a displacement field obtained from ultrasound and digital image correlation techniques [10]. For the QT, a linear elastic material was assumed; with values of $30 \mathrm{MPa}$ for Young's modulus and 0.46 for the Poisson ratio. The cartilages and bones are modelled as rigid bodies for simplicity reasons and as their impact was assumed to be minimal on the outcomes

Regarding the embedded parts, to account for the FEM voxels that are either empty or partially filled with the embedded surface mesh, the mass and the stiffness values of the embedding FEM are weighted based on the methods proposed by Nesme et al. to improve the resulting behavior of the model [6]. This technique is fully implemented in Artisynth.

For the patellar ligament and the medial and lateral retinaculum, each of their bundles was represented by a discrete number of strands. Tensile forces were computed assuming each strand behaved as a non-linear stiffening spring at low strains $(e<0.06)$ and having a linear stiffness at higher strains [11]. The patellar li
bundles consisting of three bundles for the cent
and lateral regions based on the results of the d
stiffness values were adapted from the literature
of the patellar ligament was set to $278 \mathrm{~N} / \mathrm{mm}^{2}$.
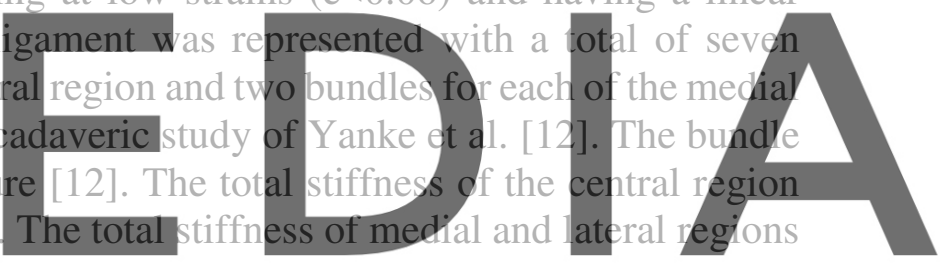
were set to $201 \mathrm{~N} / \mathrm{mm}^{2}$ and $173 \mathrm{~N} / \mathrm{mm}^{2}$ respectively. The nominal reference strain was

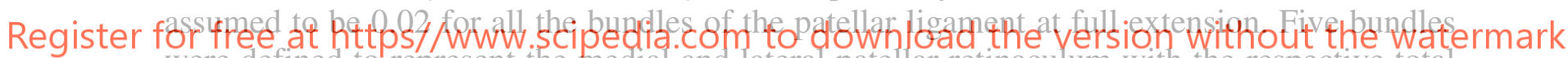
were defined to represent the medial and lateral patellar retinaculum with the respective total stiffness of $31 \mathrm{~N} / \mathrm{mm}^{2}$ and $97 \mathrm{~N} / \mathrm{mm}^{2}$ adopted from the literature $[13,14]$ while a 0.01 reference strain was assumed for them.

\subsection{Load and boundary conditions}

The passive deep knee flexion is simulated by imposing the motion of the tibiofemoral joint. As the quadriceps muscles are all connected to QT and the QT is attached to the patellar bone, changing the modeling technique of the quadriceps muscles will affect the kinematics of the patellofemoral joint that is free to react to the forces transmitted to it through the soft tissues and contact forces.

The idea is to impose the motion of the tibiofemoral joint based on the MRI segmented bones at the highest level of flexion and then to take the patellofemoral joint kinematics as the output of the simulation. This output can be directly compared to the bone positions obtained from MRI at various flexion angles and enables us to validate the simulation outcomes by comparing the patellofemoral kinematics. A schematic view of the setup used for the simulation of passive deep knee flexion is depicted in Figure 5.

As demonstrated in Figure 5, the bone positions at $25^{\circ}$ knee flexion (the first MRI position) 
are used to define the initial position of the model. The pelvic bones and femur are fixed in their initial positions. The foot bone and the fibula are constrained to the tibia and considered as a single segment. The patella is free to move in all its six Degrees of Freedom (DOFs) and its motion will be in response to the forces transmitted to it through the muscles and tendons, as well as contact forces.

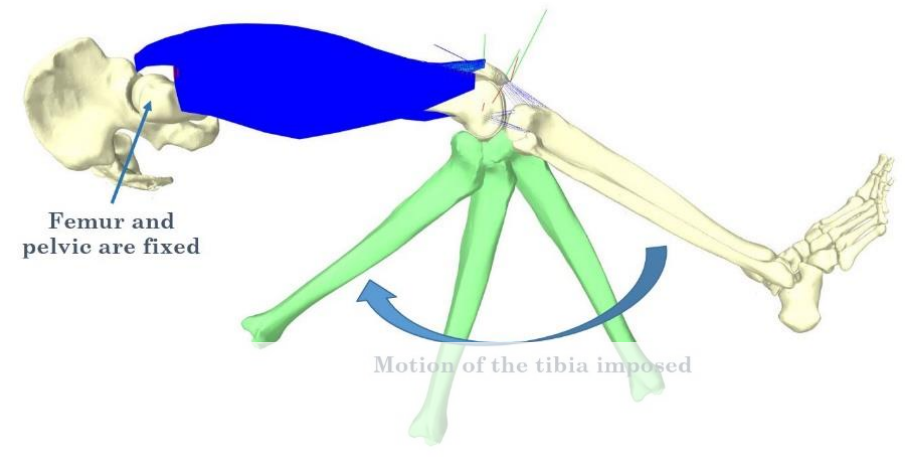

Figure 5: Schematic view of the passive deep knee simulation setup. The position of the combined shank and foot segment is controlled through the transformation matrix obtained from MRI at different knee flexion angles. The thigh and pelvic segments are non-dynamic. The patella is free to move in response to forces transmitted to it through the soft tissues and to contact with the femur.
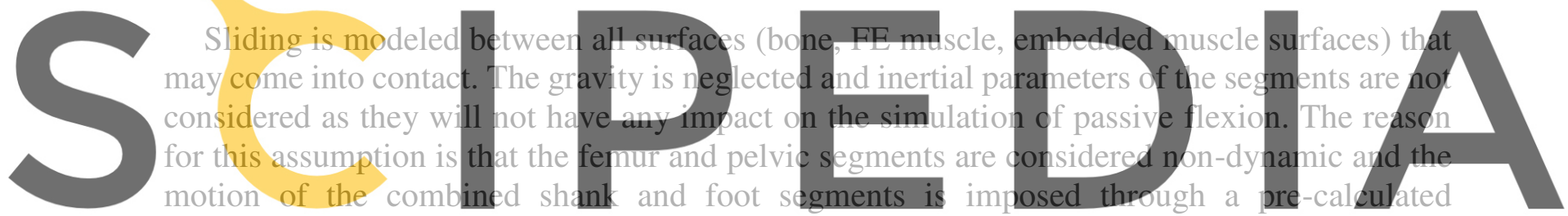

transformation matrix at each time increment.

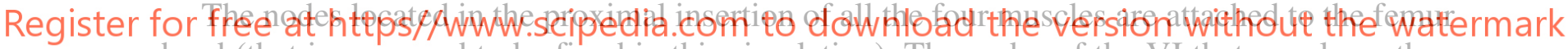
head (that is supposed to be fixed in this simulation). The nodes of the VI that are closer than one millimeter to the femur surface are attached to the femur. To represent the distal insertion of the four muscles to the QT, a distance limit with the QT is defined for each muscle, and all the nodes that are closer to the QT than the distance limit are attached to the proximal part of the QT. The nodes located in the distal part of the QT are attached to the patella.

\subsection{Model validation through comparison with MRI based kinematics}

Defining the local coordinate frames for the shank, patella, and thigh segments were required to impose the motion of the shank and to extract the kinematics of the patella. The bony landmarks and knee Joint Coordinate System (JCS) are defined based on the ISB standards $[15,16]$. For the calculation of the translations, the origins of the femur and shank local frames were both defined to be placed midpoint between the medial and lateral femoral epicondyles at neutral position (considered to be the bone positions while taking the CT scan). For the patellar segment, the origin was defined to be located on the most distal point of the patella bone. As the patellofemoral kinematics was observed in response to the passive flexion of the tibia, the tibiofemoral flexion angle was defined as the independent variable and all the 6 DOFs of the 
patella were considered to be dependent on it.

To compare the kinematic predictions of our knee models with the MRI-based data points, the Normalized Root-Mean-Square Deviation (NRMSD) from the MRI data was computed. Normalization was performed for each DOF because the scale and units are different. The sum of the normalized RSMD is computed over all 6 DOFs (Equation 6) and reported as the total NRMSD for each perturbation.

$$
N R M S D=\sum_{i=D O F} \frac{R M S D_{i}}{\bar{X}_{i}}
$$

$R M S D_{i}$ is the root-mean-square deviation of the patellar motion in its $i$ th DOF from the MRI data points in that DOF. $\bar{X}_{i}$ is the mean of the three MRI data points in the $i$ th DOF. Lower values of NRMSD mean less deviation from the MRI data points that are directly extracted from images at different flexion angles.

\section{RESULTS AND DISCUSSION}

The model of the embedded surface mesh for VI, VL, and VM muscles were successful to simulate the whole deep knee flexion. The deformed state of the full and reduced models at various steps of the simulation is depicted in Figure 6. Regarding the simulation time, the finelymeshed FE model took more than 8 hours to complete while the embedded model took about two hours and a half, despite that the Rectus Femoris and quadriceps tendon are still finely
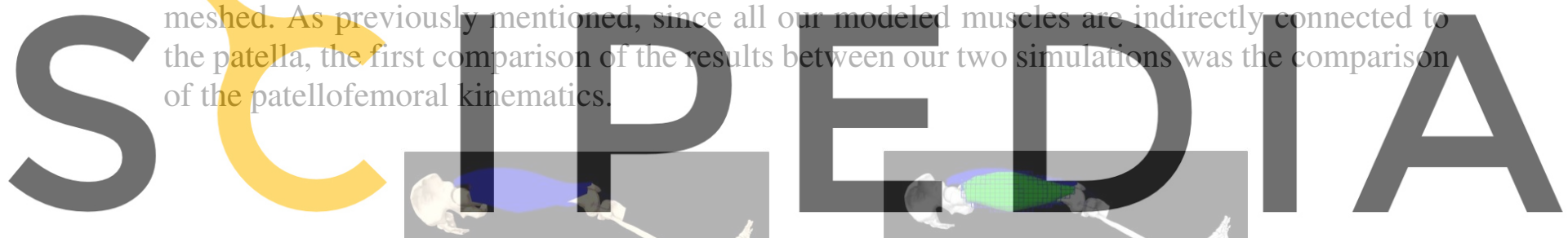

Register for free at https//www.scipedia.com to download the version without the watermark
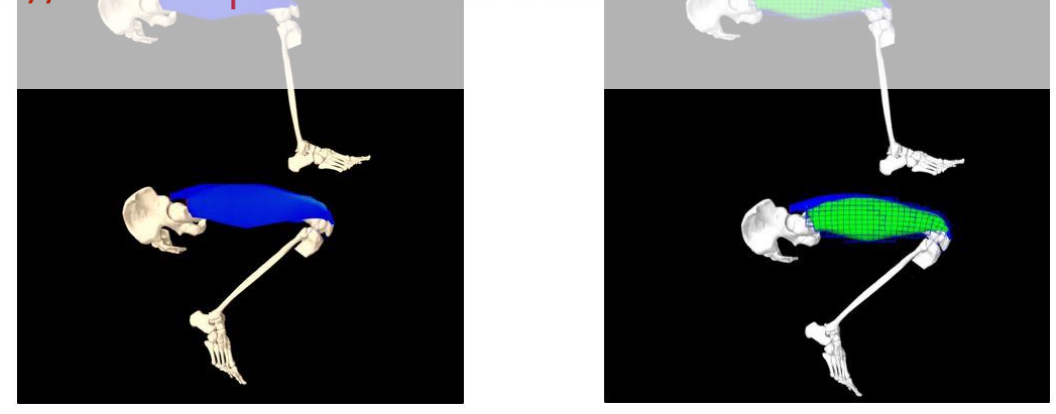

Figure 6: left: The deformed state of the finely-meshed FE model at various steps of passive knee flexion. Right: The deformed state of the model with embedded surrounding muscles at various flexion degrees.

The predicted patellofemoral kinematics are demonstrated in Figure 7 and compared with the outputs of the finely meshed FE model. The predicted kinematics by the two models are compared against MRI data points through computing the NRMSD values as presented in Table1. As the results of this table are suggesting, the patellofemoral kinematic outcomes did not substantially change and were not compromised by using the reduced model. 
To perform a preliminary investigation on the impact of changing the modeling technique of the VI, VL, and VM muscles on the predicted stress distribution of the RF muscle, we have also monitored the maximum principal stress in the RF muscle throughout the simulation time. The result is presented in Figure 8. The RF maximum principal stress was not noticeably impacted by changing the modeling technique despite the reduced computational time.
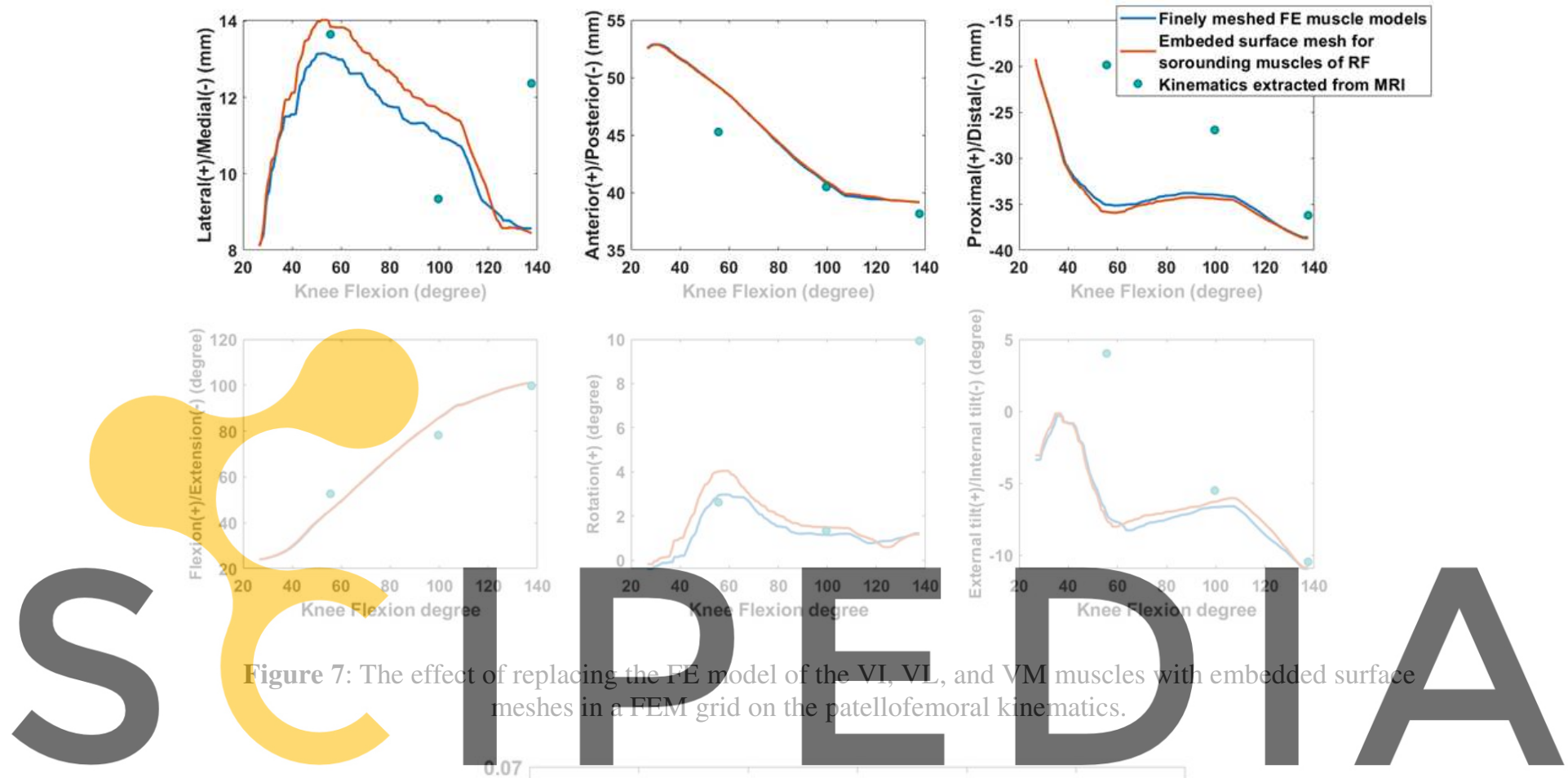

Register for free at https//www.scipedia.com to download the version without the watermark

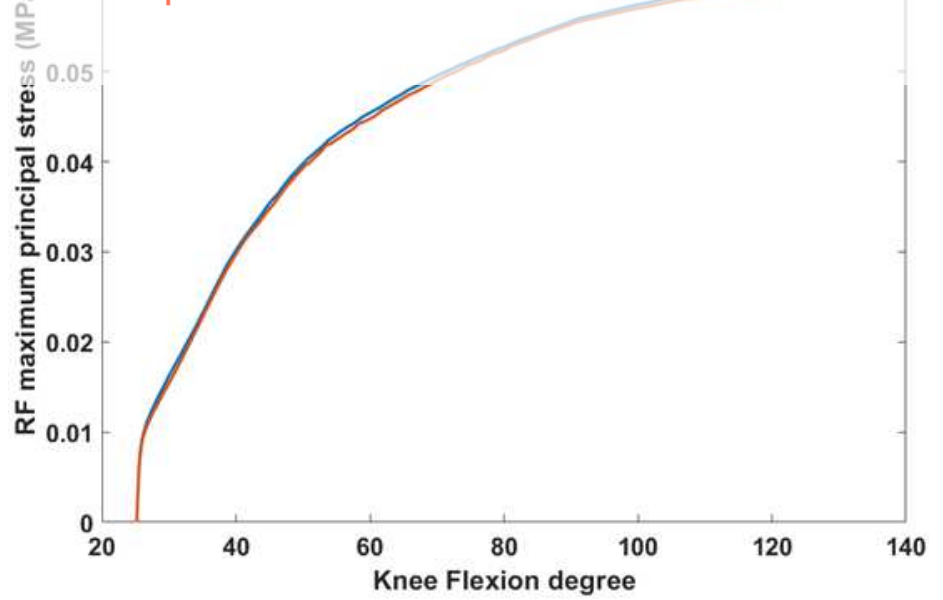

Figure 8: Maximum principal stress of the RF muscle that was considered as the main muscle throughout the passive deep knee flexion simulation with finely meshed FE models of the quadriceps muscle group and QT (blue line), and finely meshed RF and QT, but replacing the VI, VL, and VM muscles with surface meshes embedded in coarse FE grids with voxel elements (orange line). 
Table 1: NRMSD computed for each DOF of the patella from the MRI data points for different models. The sum of the deviation of all DOFs is presented in the last column.

\begin{tabular}{|l|c|c|c|c|c|c|c|}
\hline \multicolumn{1}{|c|}{ Patella DOF } & & & & & & \\
Muscle Model & Lateral & Anterior & Proximal & Flexion & Rotation & Tilt & Sum \\
\hline FE model of all muscles & 0.21 & 0.06 & 0.35 & 0.08 & 1.08 & 1.63 & 3.40 \\
\hline Embeded mesh for VI, VM and VL & 0.22 & 0.06 & 0.37 & 0.08 & 1.10 & 1.69 & 3.52 \\
\hline
\end{tabular}

\section{CONCLUSION}

The current study evaluated mesh embedding to be used as a model reduction technique in the MS models of the lower limb that represent the muscles with FE components. The full and reduced lower limb models were used to simulate passive deep knee flexion and the kinematic outcomes were compared against the MRI images of the same subject at different flexion angles. The use of the mesh embedding technique was successful to substantially reduce the computational cost of the simulation as well as model preparation time. The outcomes of the reduced model did not show a substantial difference from the full model.

\section{ACKNOWLEDGMENT}

This work was funded by Fondation pour la Recherche Médicale under the project FRM

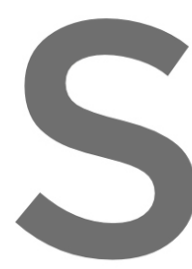
DIC20161236448. 'Th n n the during the process of this stud REFERENCES Mo F, Li F, Behr M, Xiao Z, Zhang G,
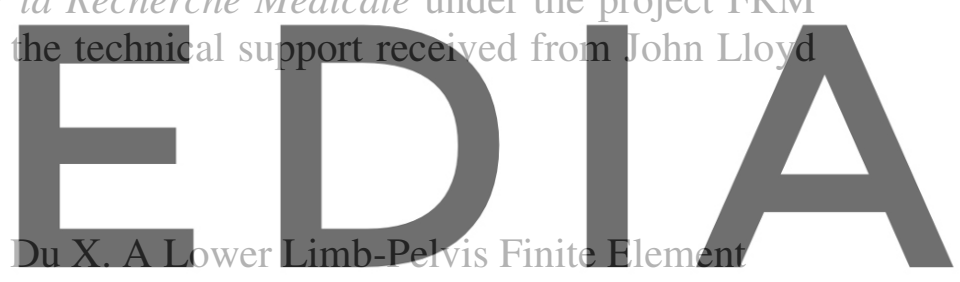

Model with 3D Active Muscles. Ann Biomed Eng 2018;46:86-96.

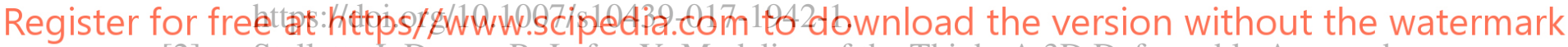

[2] Stelletta J, Dumas R, Lafon Y. Modeling of the Thigh: A 3D Deformable Approach

Considering Muscle Interactions. Biomech. Living Organs Hyperelastic Const. Laws

Finite Elem. Model., 2017, p. 497-521. https://doi.org/10.1016/B978-0-12-8040096.00023-7.

[3] Blemker SS, Pinsky PM, Delp SL. A 3D model of muscle reveals the causes of nonuniform strains in the biceps brachii. J Biomech 2005;38:657-65. https://doi.org/10.1016/j.jbiomech.2004.04.009.

[4] Blemker SS, Delp SL. Rectus femoris and vastus intermedius fiber excursions predicted by three-dimensional muscle models. J Biomech 2006;39:1383-91. https://doi.org/10.1016/j.jbiomech.2005.04.012.

[5] Siebert T, Till O, Stutzig N, Günther M, Blickhan R. Muscle force depends on the amount of transversal muscle loading. J Biomech 2014;47:1822-8. https://doi.org/10.1016/j.jbiomech.2014.03.029.

[6] Nesme M, Kry PG, Jeřábková L, Faure F. Preserving topology and elasticity for embedded deformable models, Association for Computing Machinery (ACM); 2009, p. 1. https://doi.org/10.1145/1576246.1531358.

[7] Lloyd JE, Sánchez A, Widing E, Stavness I, Fels S, Niroomandi S, et al. New 
techniques for combined FEM-Multibody anatomical simulation. Lect. Notes Comput. Vis. Biomech., vol. 999, Springer Netherlands; 2019, p. 75-92. https://doi.org/10.1007/978-3-030-23073-9_6.

[8] Lloyd JE, Stavness I, Fels S. ArtiSynth: A Fast Interactive Biomechanical Modeling Toolkit Combining Multibody and Finite Element Simulation. Stud. Mechanobiol. Tissue Eng. Biomater., vol. 11, Springer; 2012, p. 355-94. https://doi.org/10.1007/8415_2012_126.

[9] Johansson T, Meier P, Blickhan R. A finite-element model for the mechanical analysis of skeletal muscles. J Theor Biol 2000;206:131-49. https://doi.org/10.1006/jtbi.2000.2109.

[10] Affagard JS, Feissel P, Bensamoun SF. Identification of hyperelastic properties of passive thigh muscle under compression with an inverse method from a displacement field measurement. J Biomech 2015;48:4081-6. https://doi.org/10.1016/j.jbiomech.2015.10.007.

[11] Blankevoort L, Huiskes R. Ligament-bone interaction in a three-dimensional model of the knee. J Biomech Eng 1991;113:263-9. https://doi.org/10.1115/1.2894883.

[12] Yanke A, Bell R, Lee A, Shewman EF, Wang V, Bach BR. Regional mechanical properties of human patellar tendon allografts. Knee Surgery, Sport Traumatol Arthrosc 2015;23:961-7. https://doi.org/10.1007/s00167-013-2768-5.

[13] Merican AM, Sanghavi S, Iranpour F, Amis AA. The structural properties of the lateral retinaculum and capsular complex of the knee. J Biomech 2009;42:2323-9. https://doi.org/10.1016/j.jbiomech.2009.06.049.

[14] LaPrade MD, Kallenbach SL, Aman ZS, Moatshe G, Storaci HW, Turnbull TL, et al. Biomechanical Evaluation of the Medial Stabilizers of the Patella. Am J Sports Med 2018;46:1575-82. https://doi.org/10.1177/0363546518758654.

[15] Wu G, Siegler S, Allard P, Kirtley C, Leardini A, Rosenbaum D, et al. ISB recommendation on definitions of joint coordinate system of various joints for the reporting of human joint motion - Part I: Ankle, hip, and spine. J Biomech 2002;35:543-8. https://doi.org/10.1016/S0021-9290(01)00222-6.

[16] Grood ES, Suntay WJ. A joint coordinate system for the clinical description of threedimensional motions: Application to the knee. J Biomech Eng 1983;105:136-44. https://doi.org/10.1115/1.3138397. 